\section{LA PERSPECTIVA DEL SISTEMA NACIONAL/REGIONAL DE INNOVACIÓN: BALANCE Y RECEPCIÓN EN ESPAÑA ${ }^{1}$}

\author{
Mikel Olazaran \\ Beatriz Otero \\ ILCLI, Universidad del País Vasco (UPV-EHU)
}

\section{THE NATIONAL AND REGIONAL INNOVATION SYSTEM APPROACH: AN ASSESSMENT OF ITS RECEPTION IN SPAIN}

\begin{abstract}
In this paper we analyze the origin and evolution of the "National Innovation System" concept and its reception in Spain. First of all, we look at the main ideas of the National Innovation System (NIS) perspective. Then attention is focused on the "Regional Innovation System" (RIS) variant, which has undergone a great development in recent years. Special attention is devoted to the role of SMEs in innovation. In the last section, we analyze the uptake of the NIS/RIS concept in Spain based on an electronic survey made with authors who have published in that field.
\end{abstract}

KEY WORDS: Innovation system, innovation in SMEs, regional innovation policy.

\section{INTRODUCCIÓN}

En este artículo se analiza el concepto de "sistema nacional de innovación" (SNI) y especialmente su variante "sistema regional de innovación" (SRI), en base a una revisión de literatura. Posteriormente, a partir de una encuesta por correo electrónico realizada a autores españoles que han publicado en este campo, se examina la recepción dichos conceptos en España.

En el apartado segundo se revisan las principales ideas de la perspectiva del SNI: el concepto de aprendizaje interactivo, la definición de innovación y la visión sobre el papel de las instituciones. También se alude brevemente a las aportaciones de la variante "sistema sectorial de innovación" y a la interacción entre investigación científica y formulación de políticas en en proceso de surgimiento de la perspectiva del SNI.
RESUMEN : En este artículo se analiza el origen y desarrollo del concepto de sistema de innovación, así como su recepción en España. En primer lugar, se examinan las bases conceptuales de la perspectiva del "sistema nacional de innovación" (SNI). A continuación, se analiza la aplicación que se ha hecho de una de las variantes con mayor desarrollo: el "sistema regional de innovación" (SRI). Se dedica una especial atención a la problemática de la innovación en las pymes. Por último, a través de una encuesta realizada entre autores con publicaciones en este campo, se estudia la recepción y aplicación del concepto del SNI/SRI en España.

PALABRAS CLAVE: Sistema de innovación, innovación en pymes, politicas regionales de innovación.

En el apartado tercero situamos el foco en la variante "sistema regional de innovación" (SRI), que ha conocido un gran desarrollo en los últimos años, y en la relevancia dicha variante para las pequeñas y medianas empresas (pymes, mayoritarias en el tejido industrial) y para las políticas regionales. A partir de la revisión de los estudios recientes, realizados en el ámbito internacional, sobre la influencia del entorno regional en la actividad innovadora de las pymes, se consideran las características específicas de la innovación en estas organizaciones y los límites de las políticas de oferta inspiradas en el modelo de "empuje tecnológico".

En el apartado cuarto se analiza la recepción del concepto SNI/SRI en España en base a una encuesta por correo electrónico a autores que han publicado en este campo. Se consideran las aportaciones del concepto, su aplicación en el contexto español, el papel de las distintas disciplinas científicas y la influencia en las políticas públicas. 


\section{El CONCEPTO dE SISTEMA NACIONAL DE INNOVACIÓN}

La perspectiva del sistema nacional de innovación (SNI) parte de una doble premisa: el conocimiento es el principal factor de producción en la economía moderna y el aprendizaje es el proceso más importante. El aprendizaje o innovación es un proceso social, influido por el contexto institucional y cultural. Cuando los agentes que interactúan comparten unas normas o valores, o pertenecen a un mismo entorno social, el proceso de aprendizaje interactivo tendrá lugar con mayor facilidad.

La perspectiva del SNI surgió en los años ochenta de la mano de economistas evolucionistas e institucionalistas como C. Freeman, R. Nelson y B. A. Lundvall, que pusieron en cuestión los postulados ortodoxos sobre el papel del conocimiento como genérico, codificable, accesible sin coste e independiente del contexto (Freeman, 1987; Dosi et al., 1988). La perspectiva tuvo un fuerte desarrollo en la década de los noventa (Lundvall, 1992a; Nelson, 1993; Edquist, 1997), llegando a adquirir un gran peso, tanto en el ámbito académico y de investigación, como en el político, en organismos como la $\mathrm{OCDE}$, de gran importancia en la política científica y tecnológica (Sharif, 2006).

Entre los casos de sistemas nacionales de innovación que sirvieron como modelos de la importancia de los factores institucionales en el desarrollo económico pueden mencionarse los de Japón (Freeman, 1987), Alemania (Freeman, 2004, e.o. como documento interno de la OCDE en 1983), Gran Bretaña y Estados Unidos (Freeman, 2002), el surgimiento de la producción masiva en EEUU y el origen de la industria basada en la ciencia en Alemania (Nelson, 2002). La tradición escandinava ha aplicado el concepto a naciones pequeñas, industrialmente avanzadas, internacionalizadas y globalmente conectadas.

Desde mediados de los noventa, la variante del sistema regional de innovación (SRI) ha conocido un importante auge (Braczyk et al., 1996; Cooke y Morgan, 1998; Storper, 1997). También se ha trabajado en los conceptos de sistemas tecnológicos (Carlsson y Jacobsson, 1997) y sistemas sectoriales (Bresci y Maleaba, 1997). Asimismo, la literatura sobre sistemas nacionales empresariales (Whitley, 1992, 1999) y variantes del capitalismo (Hall y Soscike,
2001; Amable, 2003) también puede ser fructíferamente relacionada con la perspectiva del SNI.

En la formulación inicial del concepto de SNI en los años ochenta por parte de autores como Freeman y Lundvall, el énfasis en la importancia de los factores institucionales y políticos implicaba una crítica de las propuestas neoliberales de debilitamiento del Estado. En este sentido, los alegatos más directos a favor de objetivos como la cohesión social se encuentran en los trabajos de Lundvall y equipo.

\begin{abstract}
"... Learning and innovation are interactive processes which depend on trust and other elements of social cohesion... The production of intellectual capital (learning) is strongly dependent on social capital. To find ways of re-establishing the social capital undermined by the globalization process is a major challenge" (Lundvall et al., 2002, 225).
\end{abstract}

La definición de "innovación" propugnada por el enfoque del SNI pone en cuestión el modelo lineal dominante, caracterizado por la preeminencia de la I+D "formal", la secuencialidad y la falta de interacciones. Para la perspectiva del SNI, las innovaciones son nuevas combinaciones de conocimiento que tienen un significado económico, llevadas a cabo normalmente por las empresas. La I+D formal es uno de los inputs que intervienen en el proceso de creación y aplicación de conocimiento, pero no es el único, ni tiene por qué ser el principal. La innovación es un proceso continuo, gradual, acumulativo, relacionado con las actividades habituales de producción. Normalmente la innovación supone usos, aplicaciones o combinaciones nuevas de elementos, componentes o posibilidades existentes. Para la perspectiva de los SNI, la innovación es un proceso interactivo y sistémico, con conexiones y realimentaciones entre las distintas fases y niveles. El sistema nunca alcanza un estado de equilibrio, puesto que los procesos evolutivos son abiertos y dependientes de una trayectoria histórica (path-dependent).

Entre los elementos de un sistema de innovación pueden mencionarse los siguientes:

- La organización interna de las empresas.

- Las relaciones entre empresas.

- Las políticas públicas y el papel de los gobiernos de distintos niveles.

- El sistema financiero. 
- El sistema de I+D.

- El sistema educativo y formativo.

- Las normas y valores sociales.

Es, por tanto, necesario ir más allá del estudio del sistema de $I+D$, entendido en sentido estricto, y considerar todos los aspectos de los procesos de construcción de competencias socioeconómicas.

"... In all parts of the economy, and at all times, we expect to find on-going processes of learning, searching and exploring, which result in new products, new techniques, new forms of organization, and new markets." (Lundvall, 1992a, 8)

Las innovaciones de producto suelen ocurrir dentro de "mercados organizados", en la interacción entre productores y usuarios (Lundvall, 1992b). Estas relaciones, que van más allá de la interacción pura de mercado, se caracterizan por ciertos niveles de intercambio de información cualitativa, cooperación y confianza mutua.

"On the one hand, pure market interactions (prices and quantities only) were found incapable of transmitting qualitative information between users and producers. On the other hand, the transformation of markets into hierarchies proposed by transaction cost theory did not materialize. We proposed to focus on interactive learning rather than transactions" (Lundvall et al., 2002, 218).

Tras revisar la definición de innovación propugnada por el enfoque del SNI, es importante considerar el papel que en el mismo juegan las instituciones. La perspectiva del SNI parte de la premisa de que las instituciones tienen una gran importancia económica. La economía institucional y evolutiva presta atención a los factores que condicionan la acción humana, y rechaza el supuesto maximizador de la teoría clásica de la acción racional.

Las instituciones son conjuntos de hábitos, rutinas, reglas, normas y leyes que regulan la relación entre personas y moldean la interacción humana (Johnson, 1992, 26). Las instituciones son "modos prevalentes de hacer las cosas", modos de coordinación, de cara a obtener un rendimiento deseado en contextos donde intervienen las acciones e interacciones de distintos actores (Nelson, 2008, 2). Las instituciones reducen la incertidumbre y la búsqueda de información necesaria para la acción individual y colecti- va, y son por tanto componentes fundamentales de todo sistema social.

"Societies which differ with respect to educational and communication infrastructures, incentive systems, the ways in which conflicts between groups and classes are coped with, ways of thinking and cooperating and so on, also learn and search differently in their production organisations" (Johnson, 1992, 37).

De un modo complementario, Lundvall y colegas (2002, 220) mencionan tres dimensiones institucionales informales: el horizonte temporal de los actores (cortoplacismo frente a visión de largo plazo), la confianza y la combinación de tipos de racionalidad (racionalidad comunicativa, empleando el concepto habermasiano ${ }^{2}$, frente a racionalidad estrictamente económica).

En última instancia, como ha apuntado R. Nelson, el enfoque del SNI trata de las relaciones entre la tecnología y las instituciones sociales.

"The advance of physical technologies is the key driving force [of economic growth]... New institutions often come into the picture as changes in the modes of interaction - new ways of organizing work, new kinds of markets, new laws, new forms of collective action - that are called for as the new technologies are brought into economic use. In turn, the institutional structure at any time has a profound effect on, and reflects, the technologies that are in use, and which are being developed" (Nelson, 2002, 269).

Las instituciones o "tecnologías sociales" (relaciones, prácticas internas a una organización, relaciones interorganizacionales) no debieran verse únicamente como "constreñimientos" a la acción, sino también, frecuentemente, como capacitadores o multiplicadores de la misma, esto es, como modos efectivos de coordinación que reducen los costes de transacción en contextos donde la cooperación humana es necesaria.

Tras analizar las principales ideas en las que se basa la perspectiva del SNI (aprendizaje interactivo, innovación e importancia de las instituciones), quisiéramos aludir brevemente a la variante de "sistema sectorial", puesto que, a nuestro entender, ilustra bastante bien la visión sobre la innovación que se propugna en aquella perspectiva.

ARBOR CLXXXV 738 julio-agosto [2009] 767-779 ISSN: 0210-1963 
Un sistema de innovación sectorial es un conjunto de empresas activo en el desarrollo y fabricación de los productos y en la utilización de tecnologías dentro de un sector industrial (Breschi y Malerba, 1997). Los sectores industriales están caracterizados por un determinado "régimen tecnológico": tipo de conocimiento, condiciones de oportunidad, apropiabilidad y acumulabilidad del conocimiento, y modos de transmisión del mismo.

Breschi y Malerba han formulado algunas hipótesis interesantes sobre la relación entre el régimen tecnológico de un sector y la importancia de la proximidad. Sus análisis son particularmente interesantes en lo que respecta a sectores maduros (como la ingeniería mecánica o la máquina herramienta) caracterizados por el carácter tácito y específico del conocimiento, la importancia de las relaciones productor-usuario y la innovación como adaptación, desarrollo o mejora del producto en respuesta a las necesidades del cliente $o$ a nuevos contextos de aplicación.

"The more the relevant knowledge base is tacit, complex, and part of larger systems, and the more the sources of new knowledge are associated with interdependent (systemic) suppliers and users, the more likely geographical proximity plays a relevant role in facilitating the transmission of knowledge across agents... The more the relevant knowledge base is codifiable, codified, simple, and independent, and the more the sources of new knowledge are associated with scientific advancements and generic (non-systemic) suppliers and users, the more likely spatial proximity does not play a relevant role in permitting the transfer of relevant knowledge" (Breschi y Malerba, 1997, 143).

Tras considerar la variante sectorial del concepto de SNI, quisiéramos terminar este apartado aludiendo a la importancia de la perspectiva SNI en la política científica y tecnológica.

El concepto de SNI es el producto de una "fertilización cruzada" entre instancias académicas y políticas (OCDE) (Sharif, 2006). Las conexiones entre la comunidad epistémica que propuso el enfoque (articulado en trabajos colectivos como Dosi et al., 1988; Nelson, 1993; Edquist, 1997) y las instancias de política científica y tecnológica de la OCDE, donde algunos de estos investigadores ocuparon puestos de responsabilidad o participaron de distintas maneras, fue decisiva para la credibilidad y legitimidad del enfoque y para su adopción en distintos paises. En la interacción entre ciencia y formulación de políticas el concepto de SNI perdió rigor metodológico o teórico (esto es, se convirtió en un marco conceptual interdisciplinar, más que en una teoría en sentido estricto), pero ganó en interés, difusión y aplicabilidad práctica. Como han indicado Albert y Laberge (2007) para el caso de Canadá, el alto nivel de aceptación del concepto de SNI entre los decisores y funcionarios públicos encargados de las políticas de $I+D$ se debe al prestigio y poder simbólico de la OCDE y de su comunidad epistémica asociada, que da un halo de cientificidad al enfoque.

\section{Pymes y sistema regional de innovación}

La aplicación del concepto de SNI a nivel regional ha ganado una creciente importancia en las esferas científica y política como instrumento para analizar los procesos de innovación a dicha escala. Con el concepto de SRI se subraya la importancia de la escala regional y de los recursos específicos regionales en la innovación de las empresas y en la competitividad de las regiones.

La perspectiva de los sistemas regionales de innovación (Cooke, 1996; Storper, 1997; Cooke y Morgan, 1998; Maskell y Malmberg, 1999; Braczyk et al., 1996) enlaza con un creciente interés por la importancia del entorno regional de la innovación, especialmente para las pequeñas y medianas empresas (pymes), y con la creciente importancia de las políticas regionales que favorecen y promueven la innovación.

La perspectiva del SRI recoge elementos de la economía evolutiva, economía institucional, teorías sociales y geografía económica, integrando diferentes conceptos como "distritos industriales", "milieu innovador" o "learning region". Esta perspectiva surge en gran medida para explicar el éxito de algunas aglomeraciones, clusters o regiones industriales (Baden Wurttemberg, Emilia-Romagna, Silicon Valley).

Desde la perspectiva del SRI, la innovación se concibe como un proceso de aprendizaje interactivo dentro de la empresa y entre ésta y otras organizaciones. Se trata además de un proceso localizado, donde factores con- 
textuales específicos pueden promover los procesos de creación y aplicación de conocimiento. Se considera que la aglomeración de empresas supone un ahorro en los costes de transacción en las relaciones entre empresas y favorece las interacciones basadas en la confianza mutua y el intercambio de conocimiento no codificado.

La perspectiva de los SRI parte de la premisa de que las redes formales e informales entre agentes (empresas, gobierno, agentes de $I+D$, otros agentes del entorno) en un contexto de confianza permiten minimizar los costes de transacción, facilitando el intercambio de conocimientos tácitos de carácter innovador (Cooke, Gomez y Etxeberria, 1997). El capital social contribuye a la innovación al reducir los costes transaccionales en las relaciones entre empresas (y entre éstas y otros actores), los costes de investigación e información, los costes de negociación y decisión y los costes de implementación de políticas y programas (Maskell, 2001; OECD, 2001).

La confianza, la reciprocidad, los valores compartidos, las redes y las normas aceleran la transferencia de información y el desarrollo de nuevo conocimiento. Los "fallos del mercado" para el intercambio de conocimiento entre empresas pueden superarse si las relaciones puramente económicas son reemplazadas por acuerdos de intercambio recíprocos y estables basados en la confianza. Las redes de colaboración interempresarial (clusters, distritos industriales, milieux) son particularmente importantes en el caso de las pymes, que conforman la gran mayoría del tejido empresarial europeo (Crouch et al., 2004).

Las caracteristicas institucionales de una región y sus infraestructuras de conocimiento son importantes condiciones básicas y pueden servir de estímulo para promover las actividades innovadoras. Por ejemplo, la creación de diferentes organizaciones locales para crear "institutional thickness" es enfatizada como importante para estimular la cooperación, el aprendizaje interactivo y la actividad innovadora. La existencia de un entorno regional que favorezca la actividad innovadora es especialmente importante en el caso de las pymes, ya que éstas se encuentran más imbricadas en el entorno regional que las empresas más grandes (Fritsch, 2001; Koschatzky y Sternberg, 2000; Koschatzky y Zenker, 1999; Kauffmann y Todtling, 2002). La región es importante para el intercambio de conocimiento tácito y para la provisión de mano de obra cualificada, ambos mecanismos muy importantes en los procesos de innovación de las empresas pequeñas (Kauffmann y Todtling, 2003; Grotz y Braun, 1997).

No obstante, no todos los SRI favorecen la actividad innovadora de las empresas. Algunos déficit de los SRI que pueden dificultar la actividad innovadora de las empresas son la falta de actores regionales relevantes ("organizational thinness"), la fragmentación o la falta de colaboración en la innovación entre agentes de la región y el "lock in" institucional social y cultural (Asheim e Isaksen, 2003; Kaufmann y Wagner, 2005; Todtling y Trippl, 2005; lammarino, 2005). Estos déficit se producen en mayor media en regiones periféricas, donde prevalecen los sectores industriales tradicionales. Para evitar el "lock in" se resalta la importancia de los vínculos extraregionales (Fritsch, 2001; Koschatzky y Sternberg, 2000; Kauffman y Todtling, 2002, 2003; Arndt y Sternberg, 2000; Oinas 2000). Simmie (2002) resalta la importancia de combinar vínculos de carácter internacional con clientes con el conocimiento local por parte de proveedores, universidades y agentes de transferencia locales.

Se han realizado diferentes estudios a nivel europeo que se centran en la influencia del entorno regional en la actividad innovadora de las pymes: en Reino Unido (Vickers y North, 2000; Kalantaridis y Phelby, 1999; Freel, 2000, 2003; Freel y Harrison, 2006), Alemania (Grotz y Braun, 1997; Fritsch, 2001; Gebauer et al., 2005), Austria (Kaufmann y Todtling, 2002; Todtling y Trippl, 2004), Europa/EEUU/Japón (Hassink, 1997), Canada (Doloreux, 2003, 2004), en regiones nórdicas (Asheim y Coenen, 2005), y los estudios europeos de carácter comparativo ERIS (Sternberg, 2000) y SMEPOL (Asheim et al., 2003).

De estos estudios se derivan las siguientes conclusiones:

- La innovación es en gran medida un proceso interno interno para la mayoría de las pymes.

- La cooperación se limita en muchos casos a clientes y proveedores. Dentro de estas relaciones es muy importante la confianza mutua y se resalta que un número limitado de relaciones usuario-proveedor duraderas y selectivas son normalmente las que moldean y restringen la mayoría de las actividades de innovación de producto.

ARBOR CLXXXV 738 julio-agosto [2009] 767-779 ISSN: 0210-1963

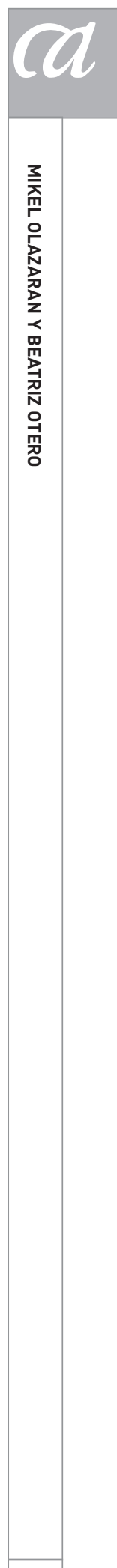

771 
- Las universidades, centros tecnológicos y otros agentes de I+D no son fuentes relevantes para la innovación. La cooperación con estos agentes se circunscribe a empresas más grandes y/o con mayores capacidades tecnológicas.

Estos estudios también muestran la existencia de capacidades específicas de la innovación en las pymes, como son:

- Base limitada de recursos, que conlleva una menor importancia de las actividades de investigación.

- Importancia del conocimiento tácito frente al codificado. El aprendizaje relacionado con las actividades productivas habituales ("learning by doing", "learning by using", "learning by interacting") juega un papel fundamental en la innovación en las pymes.

- Cultura organizacional distintiva que se deriva de la combinación propiedad/dirección. El background socioeconómico del propietario/gerente y su actitud personal (reactiva, activa o proactiva) tiene un impacto importante en el rango de fuentes tecnológicas. Se ha señalado que las pymes con un gerente de una segunda generación, especialmente aquellos que han estudiado en universidad, acuden a una mayor variedad de fuentes externas y consiguen buenos resultados del contacto con universidades.

- Menor capacidad para moldear e influenciar el entorno externo.

- Importancia de la figura del "gatekeeper" relacionada con la capacidad de absorción de la empresa.

En los años ochenta y noventa tomaron un gran impulso las políticas regionales de oferta inspiradas en la idea del empuje tecnológico ("technology push" o modelo lineal tecnológico). Estas políticas se dirigieron a fortalecer una estructura intermediaria para asegurar que las nuevas tecnologías llegaban a las empresas regionales. El objetivo era crear un entorno de innovación favorable para las empresas regionales, particularmente pymes. La intervención se centró en crear mecanismos de interfaz, centros tecnológicos y parques científicos y tecnológicos, con el objetivo de poner en contacto la demanda y la oferta tecnológica.

Bajo el modelo del "technology push", se asumía que la provisión de "inputs de I+D" o una oferta tecnológica a disposición de las empresas (o la mera financiación pública de la I+D privada) provocaría por sí misma los procesos de transferencia. No se prestó suficiente atención a las características del conocimiento tecnológico y de las organizaciones destinatarias del mismo (las pymes), así como a los múltiples tipos de interacciones que se establecen en los sistemas de innovación (Todtling y Trippl, 2005). Como resultado, las políticas de transferencia desde el lado de la oferta han alcanzado mejor a empresas grandes y/o con ciertas capacidades tecnológicas que a pymes y empresas con menores capacidades de innovación (Hassink, 1997; Kauffmann y Todtling, 2002).

En este sentido, en el ejercicio RIS ("Regional Innovation Systems") de la UE, que ayudó a diseñar políticas de innovación en muchas regiones europeas en los noventa, se encontró que la cooperación a nivel regional es clave para la innovación en las pymes, y que los partners más importantes en el proceso de innovación son, en orden de importancia: 1) clientes, 2) proveedores, 3) competidores y 4) organizaciones de apoyo (como infraestructuras de I+D) (Landabaso, 2000). Las empresas regionales, a menudo pequeñas, de propiedad familiar y competidoras entre sí en mercados relativamente cerrados, no tienen una tradición de cooperación y confianza, ni entre ellas mismas ni con las infraestructuras de I+D regional, especialmente las universidades. La cooperación para la innovación es particularmente crítica en las pymes debido a los recursos humanos internos limitados y a la falta de know how. Existe una falta de integración entre la oferta y la demanda regional de tecnología e innovación. Las empresas no expresan una demanda de innovación y las infraestructuras de I+D regionales no están imbricadas en la economía regional (son incapaces de identificar las necesidades y capacidades de innovación existentes en la economía regional).

En los últimos años las políticas de I+D van incorporando el concepto de sistema de innovación en el discurso, pero esto no siempre se traduce en nuevos instrumentos acordes con dicho concepto. En esta línea, en un estudio europeo comparativo de 40 instrumentos de la política regional de innovación en 11 regiones europeas, los autores indican que la mayoría de las herramientas pueden ser etiquetadas como "relativamente convencionales", es decir, no tienen en cuenta la existencia de mecanismos interactivos que pueden propiciar los procesos de aprendizaje, "adicionalidad" y networking. En el estudio se señala 
que en la implementación de las políticas no se han tenido en cuenta las reflexiones teóricas derivadas de los estudios de los sistemas locales/regionales de innovación (Garofoli y Musyck, 2001; Nauwelaers y Wintjes, 2002).

\section{Recepción del concepto de SNI/SRI en España}

El concepto de SIN/SRI fue recibido en España en los años ochenta y comienzos de los noventa, concidiendo con la formulación de las primeras políticas científicas y la recepción de la perspectiva de los estudios sobre ciencia, tecnología y sociedad (Luján, López-Cerezo y Muñoz, 1994; Sanz, 1998; Olazaran y Moso 1999). Investigadores de economía/empresa, sociología/ciencia política y filosofía centraron su foco de interés en la ciencia y la tecnología. Durante unos años la comunicación interdisciplinar entre estos investigadores tuvo lugar en los congresos de la red RICTES, hasta que esta red cesó su actividad a finales de los noventa. Recientemente, los investigadores pertenecientes a distintos institutos deI CSIC han sido agrupados en una estructura de coordinación denominada red CTI-CSIC pero, en general, puede decirse que la integración interdisciplinar de este campo en España es mucho menor que en otros países europeos.

Tras el pico inicial de interés, que puede verse como una "moda académica" (Abrahamson, 1996), los estudios sobre la innovación se realizan con "normalidad" (aunque con un nivel de actividad e institucionalización menor que en otros países) en las distintas disciplinas y centros. Debido a los límites de este artículo, mencionaremos sólo algunos grupos o centros representativos. En economía/empresa pueden mencionarse, entre otros, los grupos representados por Xavier Vence (USC), Mikel Buesa, José Molero (IAIF, UCM), Ignacio Fernandez de Lucio (CSIC-UPV, Valencia), Mikel Navarro (UD), Mikel Gómez Uranga (UPV-EHU), Clara Eugenia García, Aurelia Modrego (UC3M), Isabel Busom (UAB) o Pere Escorsa (UB). En sociología/ciencia política cabe mencionar los grupos de Luis Sanz (IPP, CSIC, Madrid), Manuel Pérez Yruela (IESA-CSIC, Córdoba), Cristóbal Torres (UAM), Teresa González de la Fe (ULL), Rubén Blanco (UCM) o Manuel Castells (UOC), así como nuestro propio grupo en la UPV-EHU. En filosofía/historia pueden mencionarse los grupos de Emilio Muñoz (CSIC), Miguel Ángel Quintanilla (USAL) y Javier Echeverría (CSIC). En estudios bibliomé- tricos, hay que mencionar el grupo CINDOC del CSIC. Por otra parte, la fundación COTEC es un centro importante de información y análisis.

En cuanto a publicaciones, mencionar siquiera una selección de las mismas está fuera de los límites de este artículo. Compilaciones interdisciplinares en castellano como Durán (1999), Olazaran y Gómez-Uranga (2001), Vence (2007) y Sebastián y Muñoz (2006) recogen aportaciones de algunos de los grupos mencionados. Revistas como Madritd, Revista d'Universitats, Recerca y Societat de la Informació, Ekonomiaz o Economía Industrial publican regularmente estudios sobre innovación, pero no existe un espacio interdisciplinar de comunicación especializado en el campo.

Para el análisis del concepto en España hemos realizado una encuesta a autores con una o más publicaciones en este campo. La selección de estos autores se ha realizado a través de la búsqueda de artículos científicos en los que se utiliza dicho concepto a partir de bases de datos de publicaciones nacionales e internacionales ${ }^{3}$. La encuesta se ha realizado vía e-mail y se han recibido 25 respuestas, entre las que están las procedentes de los autores que más han trabajado el concepto en España desde las ciencias económicas y sociales.

\subsection{Aportaciones del concepto}

Los expertos encuestados destacan dos aportaciones principales del concepto de sistema nacional de innovación: el carácter sistémico e interactivo de los procesos de creación de conocimiento, superando el modelo lineal, y la interrelación entre actores y componentes, por un lado, y la importancia del contexto espacial, social e institucional de la innovación, por otro. También se señala su utilidad para redefinir las políticas de $I+D+i$, al menos a nivel del discurso (la implementación, como se verá más adelante, ha sido bastante más limitada). Algunas personas señalan que se trata de un marco conceptual, de tipo heurístico más que estrictamente teórico, que facilita la recogida y organización de información, la comparación y el diálogo entre disciplinas.

"A mi entender la aportación del concepto de NIS es la siguiente: a) Visión de conjunto de los procesos de innovación en el ámbito territorial definido analíticamente. b) Perspectiva institucional y organizativa en el estudio de los procesos 
de innovación. c) Posibilidad de identificar 'gaps' o problemas en el sistema, más allá de los errores convencionales del mercado. d) Visión de la dimensión evolutiva de los procesos de innovación, entendiendo que hay muchos elementos de incertidumbre y procesos. e) Entender que los procesos de innovación son altamente influidos por el contexto socioeconómico en el que están embebidos." (E3)

"A mi entender la principal aportación es el haber hecho ver, tanto a los estudiosos de estos procesos como a los responsables de las politicas científicas y de innovación, la importancia de las relaciones entre los agentes y de las condiciones de contexto (leyes, normas, usos y costumbres, etcétera) en los procesos de innovación." (E6)

"El concepto sirvió para dotar a los estudios sobre innovación tecnológica de una mayor coherencia, y permitió que los análisis realizados desde perspectivas disciplinares muy distintas pudiesen converger en el uso de conceptos, marcos teóricos, factores y variables para el análisis. En esta primera etapa dicho concepto también facilitó la adopción de un lenguaje común entre investigadores dedicados al estudio de la innovación, los responsables de las políticas de $\mathrm{I}+\mathrm{D}+\mathrm{i}$ y los propios actores empresariales." (E11)

\subsection{Aplicación en España}

De un modo muy mayoritario, las personas entrevistadas subrayan que la aplicación del concepto de SNI en España ha tenido lugar a través de su variante regional (sistema regional de innovación, SRI), tanto a nivel de análisis como de definición de políticas, en concordancia con la dinámica territorial de los procesos de innovación. Los estudios a nivel regional se han visto favorecidos por la estructura autonómica y por la concentración industrial en algunas regiones. Algunos expertos señalan expresamente que no ha habido una aplicación del concepto a escala nacional.

Asimismo, se han recogido algunas opiniones críticas respecto a un excesivo énfasis en las políticas públicas y sobre la falta de indicadores sobre las relaciones entre actores.

"Esto en un país como España, donde las legislaciones autonómicas empezaron a diferenciar las actuaciones y donde la actividad industrial estaba muy concentrada, empezó a dar origen a diversos sistemas regionales. La herramienta conceptual se reveló muy eficaz para reflejar la realidad." (E17)
"Creo que la variante denominada Sistema Regional de Innovación se ha aplicado y sigue aplicando de forma excesiva en España como resultado de la 'venta' que tiene las distintas estancias de las Administraciones Autonómicas y que financian generosamente los trabajos que llevan esta etiqueta. También creo que las aplicaciones que se han hecho en nuestro país han estado dominadas por las dimensiones políticas y públicas del concepto, en detrimento de un mayor énfasis por el comportamiento empresarial. En mi modesta opinión, creo que en España sí que hay auténticos sistemas regionales de innovación, con orígenes bien definidos, lo que no creo es que haya tantos sistemas regionales de innovación como gobiernos autonómicos. Este tipo de estudios parte ya de un a priori que no responde al verdadero concepto de Sistema de Innovación: el hecho de que las políticas públicas son las que vertebran y alumbran los sistemas de innovación. La situación político-administrativa es una variable más." (E11)

"En general creo que se ha abusado del concepto o de la 'metafora', y que a veces se ha dado por sentada la existencia de un 'sistema' sin comprobar empíricamente la presencia de sus elementos y de sus propiedades sistémicas. Se echa en falta en la literatura una distinción analítica más clara entre las 'políticas', y el 'sistema', sobre todo a la hora de explicar unas u otro, o de hacer atribuciones causales entre ambos." (E7)

"En España se ha aplicado básicamente al diagnóstico de los sistemas (nacional, regionales, sectoriales,...) pero, a la hora de la verdad, se han seguido analizando los diferentes subsistemas o entornos (científico, productivo...), cada uno por su lado, porque las relaciones siguen sin ser bien estudiadas debido a la falta de indicadores fiables y universales sobre ellas. En los datos del INE sólo se informa desde el punto de vista cuantitativo sobre los flujos financieros entre empresas y universidades y entidades de las AAPP, pero eso no informa sobre la importancia de las relaciones. En la encuesta de innovación tecnológica hay dos preguntas cualitativas que ayudan: con quién coopera la empresa y la importancia de diversas fuentes de información sobre la innovación, pero son datos cualitativos. En bases de datos de patentes se informa sobre los artículos y patentes citadas, pero en gran medida esa información se usa para delimitar la aportación (inventiva) del solicitante, no para informar sobre las colaboraciones. En fin, como digo, mientras no se tenga más y mejor información sobre las relaciones será difícil que el concepto de sistema pase de ser eso, un concepto, para ser una teoria. Yo diria, pues, que, en definitiva, ha sido una 
aproximación conceptual muy útil desde el punto de vista intelectual, pero la noción de sistema apenas se ha aplicado más que de una forma superficial, por falta de indicadores para medir aquello que supuso la verdadera aportación: las relaciones." (E6)

\subsection{Disciplinas en España}

En cuanto al papel de las distintas disciplinas científicas, destaca el papel principal de la economía aplicada, la economía regional y la economía de la innovación y, en segundo lugar, la contribución de politólogos (analistas de políticas públicas) y sociólogos de la ciencia. También hay una mención a la organización de empresas, la geografía regional o local, la cienciometría y la filosofía. No obstante, se señala que los economistas han aportado menos que en otros países, y que las relaciones entre disciplinas son muy escasas.

"Los grupos más activos en el desarrollo del concepto en España proceden de la economía (especialmente la economía aplicada), las ciencias empresariales, la sociología, la cienciometria y la filosofía. La influencia de investigadores que han pasado por el mundo de la gestión universitaria ha sido importante. Excepto por esfuerzos aislados, no existe una masa crítica interdisciplinar que aborde sistemáticamente el concepto." (E13)

"No es un concepto que haya calado especialmente en la Economía, en especial en la economía pública que es donde tendría que haber calado. Más bien ha quedado en manos de los 'policy makers', las instituciones que crean informes (Ministerio, CDTI, COTEC, etc.), en especial las de algunas comunidades autónomas, donde no sólo usaron el concepto sino que lo aplicaron. Un buen ejemplo es la SPRI en el País Vasco. Quiero decir con esto que el debate como mucho académicamente ha quedado en las reuniones de los centros que se dedican a Estudios de Ciencia y Tecnología, que suelen ser pluridisciplinares." (E17)

"Creo que los economistas han aportado a este tema menos que en otros países, en gran medida porque es un tema donde los modelos formales, que es lo único que interesa a la mayoría de colegas, no es fácil." (E10)

"Son los economistas y los politólogos los que más han utilizado el concepto, tomado generalmente como marco o teoría sin hacer aportaciones críticas. No ha habido relación entre disciplinas o subdisciplinas, como generalmente suele ocurrir en España." (E19)
"Los economistas han tratado de describir y analizar los sistemas regionales de manera cuantitativa y los sociólogos el comportamiento de las instituciones y las políticas. No ha habido mucha relación entre economistas y sociólogos." (E9)

"Aunque el marco impulsa a una aproximación multidisciplinar, tengo la sensación de que cada uno sigue abordando el análisis desde lo que constituye su área de conocimiento original (economía, sociología...)." (E20)

\subsection{Influencia en las políticas}

Los entrevistados destacan la influencia del concepto en la definición de las políticas, sobre todo regionales, pero no tanto en lo que se refiere a la implantación de las mismas. Existe, por tanto, un riesgo de utilización de un discurso de sistema de innovación (influido por las instancias internacionales) para legitimar políticas que en realidad son más lineales o que responden a intereses existentes. Por otro lado, algunos entrevistados señalan que la interacción entre el mundo académico y político ha sido menor en España que en otros países. También se apuntan las dificultades para coordinar las distintas partes del sistema (administraciones públicas entre sí; sector público y empresa), la rigidez del sistema público y la falta de una cultura de innovación y transferencia de conocimiento en la empresa. Los instrumentos que favorecen la cooperación (proyectos), así como las estructuras de interfaz (como centros tecnológicos), son mejor valoradas por algunas de las personas entrevistadas.

"Eso se debe en parte a la utilización de los conceptos de sistema nacional/regional de innovación en la doctrina comunitaria, que ha reforzado y legitimado hacia abajo este tipo de conceptualizaciones." (E24)

"Creo que el impacto de la adopción del concepto ha sido muy fuerte a nivel, como ya he indicado, del 'marketing' o 'venta' de las distintas actuaciones e instrumentos, pero no creo que las políticas públicas realmente reflejen el concepto, ya que siguen persistiendo en su diseño e implementación factores de segregación y una clara falta de integración en aspectos críticos. La mejor muestra de ello creo que se observa en la clara contradicción existente en la gestión de los recursos humanos de Universidades y OPIs y al mismo tiempo el pretendido fomento de la cooperación entre universidad y empresa." (E11)

ARBOR CLXXXV 738 julio-agosto [2009] 767-779 ISSN: 0210-1963

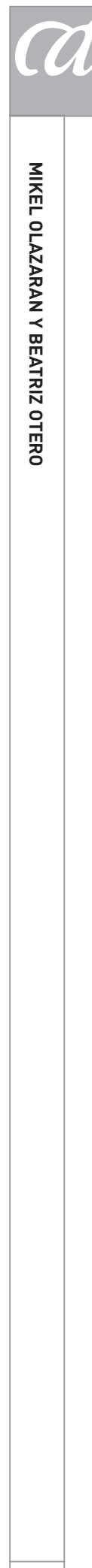

775 
"La incidencia ha sido limitada, creo que ha calado en el discurso político, pero que no se ha trasladado tanto a los instrumentos, que, sobre todo a nivel nacional, siguen estando muy centrados en las políticas directas, basadas en la transferencia de recursos. Quizás en alguna región sí se ha experimentado con instrumentos que se dirigen más a fomentar las redes, las interacciones y el aprendizaje." (E7) "La incidencia ha sido prácticamente nula. Como repito continuamente hay una gran distancia entre el discurso y la acción en políticas científicas y de innovación.Todas las convocatorias desde hace veinte años en España están inspiradas en el modelo lineal." (E19)

"En todo caso, en la aplicación y diseño de las políticas abunda más el vocabulario de los SRI que una verdadera concepción sistémica de todo el proceso y una auténtica articulación de las diferentes patas de las políticas que inciden en la dinámica de la innovación" (E24)

"El concepto ha contribuido a legitimar el papel de las instituciones que ponen en contacto los distintos elementos del sistema, tanto organizaciones (OTRI-OTT, FUE, CEEI, etc.), como programas públicos de financiación (PROFIT, PETRI, CENIT) o de incorporación de científicos en la empresa (Programa Torres Quevedo)... También ha contribuido a aumentar la conciencia sobre los procesos de evaluación de las actividades de investigación (SISE, COSEP, CNEAI)." (E13)

"En Suecia y en los paises nórdicos en general ha habido una conexión estrecha entre el ámbito académico y político. En otros países esto es discutible, por ejemplo en España." (E18)

"Desconozco si podriamos hablar de una co-evolución entre las dos esferas, la de corte más científico y la más política. En otros países sí que van de la mano, pero en éste no lo creo." (E25)

\subsection{Debilidades del concepto de SNI/SRI}

Los entrevistados señalan algunas debilidades del concepto que señalamos a continuación. Se señala que se trata de un concepto descriptivo y que existe una carencia de indicadores para desarrollar un marco analítico. Los entrevistados señalan que la falta de indicadores ha dado lugar a una literatura ambigua e imprecisa.

"Creo que se ha avanzado en la descripción, pero queda todavía quehacer en el terreno de obtención de datos para elaborar indicadores en diversos ámbitos (inputs, outputs, instituciones) y en el del contraste empírico de las relaciones entre los mismos, tanto a nivel agregado como microeconómico." (E4)

"En este sentido, por lo que respecta a la parte científica del concepto, le veo muchas carencias, ya que hay una gran cantidad de aspectos que no se han podido estudiar todavía en detalle (como los indicadores, los métodos de evaluación, el grado de interacción en un sistema y su repercusión sobre el nivel de desarrollo del mismo, etc.) y que creo que debilitan al concepto." (E25)

"Ciertamente la literatura de sistemas regionales de innovación es claramente ambigua e imprecisa, y con poco contraste empírico." (E20)

Por otro lado, se indica que se ha hecho un uso abusivo del concepto que ha llevado a que éste haya perdido parte de su validez analítica y descriptiva. Como señala un entrevistado, paradójicamente la adopción del concepto por parte de los decisores políticos ha supuesto un cierto debilitamiento en la vertiente científica.

"Asi pues, de la noche a la mañana a las políticas de ciencia o tecnológicas, se les ha puesto la coletilla de innovación, pensando que con ello ya se crea un sistema. Es más, en muchas regiones españolas, se habla de sistema regional, aún y cuando todos sepamos o por lo menos deberiamos saber que un sistema regional conlleva otras muchas connotaciones. En este sentido, no creo que podamos hablar de sistemas regionales de innovación consolidados a nivel regional en España... Son pocas las regiones que podrian considerarse como acreedoras de un sistema regional de innovación consolidado. Sin embargo, todas ellas hablan de poseer un sistema. Este hecho creo que le favorece poco al concepto, así como a su uso." (E25)

\section{Conclusiones}

La idea central de la perspectiva del SNI es que las personas, las organizaciones y las instituciones, esto es, las relaciones sociales, juegan un papel importante en los procesos de innovación, que van por tanto más allá de las relaciones meramente económicas caracterizadas por la acción racional instrumental. El concepto de SNI ha supuesto una nueva concepción de la innovación que supera el modelo lineal y que se adapta mejor a la realidad de la innovación en las empresas, sobre todo pymes. Asimismo, 
ha proporcionado un marco para el estudio de variables sociales en el ámbito de la innovación.

El concepto ha mostrado su potencialidad para analizar procesos de innovación a escala regional y, en concreto, los factores del contexto (sociales, institucionales, culturales y políticos) que pueden influir en la actividad innovadora de las empresas. En este sentido, los estudios realizados a nivel internacional muestran la imbricación de las pymes en entornos regionales, así como la especificidad de la innovación que realizan. El concepto también es útil en la definición de políticas, aunque no se ha avanzado tanto en su implementación. Las políticas regionales para fomentar la innovación en pymes han mostrado sus límites, en parte por ceñirse en gran medida al modelo lineal de creación de una oferta tecnológica.

De la encuesta realizada sobre la recepción y desarrollo de la perspectiva SNI/SRI se deduce que en España la aplicación del concepto ha sido fundamentalmente a nivel regional. Aunque se señalan las virtudes del concepto para analizar los procesos de innovación, también se apunta a sus limitaciones, entre las que destacan la falta de indicadores para el análisis empírico de las relaciones entre actores, la falta de estudios comparativos y el uso abusivo del concepto en el discurso político, que no se ha correspondido con una implementación efectiva en el diseño de políticas de innovación.

\section{NOTAS}

1 Este trabajo fue realizado dentro del proyecto "Cooperación, conocimiento e innovación en la industria española de máquina herramienta" financiado por el Ministerio de Educación y Ciencia. SEJ2005-06075

2 Véase Habermas, 1987 (v.o. 1981).

3 La búsqueda se ha realizado en las siguientes bases: ISOC/CSIC, Dialnet, Abi Inform, Ebsco, Ingenta y Science Direct con los siguientes descriptores: "sistema de innovación" (en las bases nacionales) e "innovation system" y Spain/Spanish/ y los nombres de las diferentes regiones españolas (en las bases internacionales).

\section{BIBLIOGRAFÍA}

Recibido: 20 de junio de 2008

Aceptado: 10 de septiembre de 2008
Abrahamson, E. (1996): "Management fashion", Academy of Management Review, vol. 21, n. ${ }^{\circ} 1$, pp. 254-285.

Albert, M. y Laberge, S. (2007): "The legitimation and dissemination processes of the innovation system approach - The case of the Canadian and Quebec science and technology policy",
Science, Technology \& Human values, vol. 32, n. 2 , pp. 221-249.

Amable, B. (2003): The diversity of modern capitalism, Oxford, Oxford University Press.

Arndt, O. y Sternberg, R. (2000): "Do Manufacturing Firms Profit from Intraregional Innovation Linkages? An Empirical Based Answer", European Planning Studies, vol. 8, n. ${ }^{\circ} 4$, pp. 465-485.

Asheim, B. y Coenen, L. (2005): "Knowledge bases and regional innovation systems: comparing nordic clusters", Research policy, vol. 34, n. ${ }^{\circ} 8$, pp. 1173-1190.

Asheim, B. T. y Isaksen, A. (2003): "SMEs and the regional dimension of innovation", en B. Asheim, A. Isaksen, C. Nauwelaers y F. Tödtling (eds.), Regional innovation policy for small-medium enterprises, Cheltenham, Edwar Elgar, pp. 21-46.

Asheim. B.; Isaksen, A.; Nauwelaers, C. y Tödtling, F. (eds.) (2003): Regional innovation policy for small-medium enterprises, Cheltenham, Edwar Elgar.

Braczyk, H.; Cooke, P. y Heidenreich, R. (eds.) (1996): Regional Innovation Systems. The Role of Governances in a Globalized World, London, University College London Press. 
Breschi, S. y Malerba, F. (1997): "Sectoral innovation systems: Technological regimes, shumpeterian dynamics, and spatial boundaries", en C. Edquist (ed.), Systems of Innovation: Technologies, Institutions and Organisations, London, Pinter, pp. 130-156.

Carlsson, B. y Jacobsson, S. (1997): "Diversity creation and technological systems: a technology policy perspective", en C. Edquist (ed.), Systems of Innovation: Technologies, Institutions and Organisations, London, Pinter, pp. 266-294.

Cooke, P. (1996): "Regional innovation systems: an evolutionary approach", en H. Braczyk, P. Cooke, R. Heidenreich (eds.), Regional Innovation Systems. The Role of Governances in a Globalized World, London, University College London Press, pp. 1-18.

Cooke, P.; Gómez, M. y Etxebarria, G. (1997): "Regional Innovation Systems: Institutional and Organizational Dimensions", Research Policy, vol. 26, n. ${ }^{\circ}$ 4-5, pp. 475-491.

Cooke, P. y Morgan. K. (1998): The associational economy: Firms, regions and innovation, Oxford, Oxford University Press.

Crouch, C.; Le-Galés, P.; Trigilia, C. y Voelzkow, H. (2004): Changing Governance of Local Economies: Responses of European Local Production Systems, Oxford, Oxford University Press.

Doloreux, D. (2003): "Regional innovation systems in the periphery: the case of Beauce in Quebec (Canada)", International Journal of innovation management, vol. 7, n. ${ }^{\circ}$ 1, pp. 67-94.

Doloreux, D. (2004): "Regional innovation systems in Canada: a comparative study", Regional Studies, vol. 38, n. ${ }^{\circ}$ 5, pp. 479-492.

Dosi, G.; Freeman, C.; Nelson, R. R.; Silverberg, G. y Soete, L. (eds.) (1988): Technological Change and Economic Theory, London, Pinter.
Durán, A. (coord.) (1999): Geografía de la innovación, Madrid, Libros de la Catarata.

Edquist, C. (1997): Systems of Innovation: Technologies, Institutions, and Organizations, London, Pinter.

Freel, M. S. (2000): "Strategy and structure in innovative manufacturing SMEs: the case of an English Region", Small Business Economics, vol. 15, n. ${ }^{\circ}$, pp. 27-45.

Freel, M. S. (2003): "Sectoral patterns of small firm innovation, networking and proximity", Research Policy, vol. 32, n. ${ }^{\circ}$ 5, pp. 751-770.

Freel, M. S. y Harrison, R. T. (2006): "Innovation and cooperation in the small firm sector: Evidence from 'Northern Britain'", Regional Studies, vol. 40, n. ${ }^{\circ}$ 4, pp. 289-305.

Freeman, C. (1987): Technology, Policy, and Economic Performance: Lessons from Japan, London, Pinter Publishers.

Freeman, C. (2002): "Continental, national and sub-national innovation systems-complementarity and economic growth", Research Policy, vol. 31, n. ${ }^{\circ}$ 2, pp. 191-211.

Freeman, C. (2004): "Technological infrastructure and international competitiveness", Industrial and Corporate Change, vol. 13, n. 3 , 531-539, e.o. 1983.

Fritsch, M. (2001): "Co-operation in regional innovation systems", Regional Studies, vol. 35, n. ${ }^{\circ}$, pp. 297-307.

Garofoli, G. y Musyck, B. (2001): "Innovation policies for SMEs in Europe: Towards an Interactive Model?", Regional Studies, vol. 35, n. ${ }^{9}$, pp. 869872.

Gebauer, A.; Woon Nam, C. y Parsche, R. (2005): "Regional technology policy and factors shaping local innovation networks in small German cities", European Planning Studies, vol. 13, n. ${ }^{\circ}$, pp. 661-683.
Grotz, R. y Braun, B. (1997): "Territorial or transnational networking: spatial aspects of technology oriented cooperation within the German mechanical Engineering Industry", Regional Studies, vol. 31, n. ${ }^{\circ}$ 6, pp. 545-557.

Habermas, J. (1987): The Theory of Communicative Action, vol. 2, Cambridge, Polity Press, 1987 (e.o. 1981).

Hall, P. y Soscike, D. (eds.) (2001): Varieties of Capitalism. The institutional foundations of comparative advantage, Oxford, Oxford University Press,

Hassink, R. (1997): "Technology transfer infrastructures: some lessons from experiences in Europe, the US and Japan", European Planning Studies, vol. 5, n. ${ }^{\circ}$, pp. 351-370.

lammarino, S. (2005): "An evolutionary integrated view of regional systems of innovation: concepts, measures and historical perspectives", European Planning Studies, vol. 13, n. ${ }^{\circ}$, pp. 497-518.

Johnson, B. (1992): "Institutional learning", en B. Lundvall (ed.): National systems of innovation, London, Pinter, pp. 23-44.

Kalantaridis, C. y Phelby, J. (1999): "Processes of innovation among manufacturing SMEs: the experience of Bedfordshire", Entrepreneurship \& Regional Development, vol. 11, n. ${ }^{\circ}$, pp. 57-78.

Kaufmann, A. y Tödtling, F. (2002): "How effective is innovation support for SMEs? An analysis of the region of Upper Austria", Technovation, vol. 22, n. ${ }^{\circ}$ 3, pp. 147-159.

Kaufmann, A. y Todtling, F. (2003): "Innovation pattern of SMEs", en B. Asheim, A. Isaksen, C. Nauwelaers y F. Tödtling (eds.): Regional innovation policy for small-medium enterprises, Cheltenham, Edwar Elgar, pp. 78-115.

Kaufmann, A. y Wagner, P. (2005): "EU regional policy and the stimulation 
of innovation: the role of European Regional Development Fund in the objective 1 Region Burgenland", European Planning Studies, vol. 13, n. ${ }^{\circ}$ 4, pp. 581-599.

Koschatzky, K. y Sternberg, R. (2000): "R\&D cooperation in innovation systemssome lessons from the European Regional Innovation Survey (ERIS)", European Planning Studies, vol. 8, n. ${ }^{\circ} 4$, pp. 487-501.

Kosckatzky, K. y Zenker, A. (1999): "The regional embeddedness of small manufacturing and service firms: regional networking as knowledge source for innovation?", Working Papers Firms and Regions N. ${ }^{\circ}$ R2/1999, Fraunhofer Institute systems and innovation research.

Landabaso, M. (2000): "Innovation and regional development policy", en F. Boekema, K. Morgan, S. Bakkers y R. Rutten (eds.), Knowledge, innovation and economic growth. The theory and practice of learning regions, Cheltenham, Edwar Elgar, pp. 73-94.

Luján, J. L.; López Cerezo, J. A. y Muñoz, E. (1994): "STS Studies in Spain: A Case Study in STS Transfer", Technoscience, vol. 7, n. 22, pp. 14-16.

Lundvall, B. (1992a): National Systems of Innovation: Towards a Theory of Innovation and Interactive Learning, London, Pinter.

Lundvall, B. (1992b): "User-producer relationships, national sytems of innovation and internationalization", en B. Lundvall (ed.): National systems of innovation, London, Pinter, pp. 45-67.

Lundvall, B.; Johnson, B.; Andersen, E. y Dalum, B. (2002), "National systems of production, innovation and competence building", Research Policy, vol. 31, n. ${ }^{\circ}$ 2, pp. 213-231.

Maskell, P. (2001): "Social capital, innovation and competitiveness", en S. Baron, J. Field y T. Schuller (Eds.), Social
Capital: Critical perspectives, Oxford, Oxford University Press, pp. 111-123.

Maskell, P. y Malmberg, A. (1999): "Localised learning and industrial competitiveness", Cambridge Journal of Economics, vol. 23, n. ${ }^{\circ}$, pp. 167-186.

Nauwelaers, C. y Wintjes, R. (2002): "Innovating SMEs and Regions: The Need for Policy Intelligence and Interactive Policies", Technology Analysis \& Strategic Management, vol. 14, n. ${ }^{\circ}$, pp. 201-215.

Nelson, R. R. (ed.) (1993): National Innovation Systems: A Comparative Analysis, Oxford, Oxford University Press.

Nelson, R. R. (2002): "Technology, institutions, and innovation systems", Research Policy, vol. 31, n. ${ }^{\circ}$, pp. $265-$ 272.

Nelson, R. R. (2008): "What enables rapid economic progress: What are the needed institutions?", Research Policy, vol. 37, n. ${ }^{\circ}$, pp. 1-11.

OECD (2001): The Well-being of Nations, The Role of Human and Social Capital, Paris, OECD.

Oinas, P. (2000): "Distance and learning: does proximity matter?", en F. Boekema, K. Morgan, S. Bakkers y R. Rutten (eds.): Knowledge, innovation and economic growth. The theory and practice of learning regions, Cheltenham, Edwar Elgar, pp. 57-69.

Olazaran, M. y Gómez-Uranga, M. (coords.) (2001): Sistemas regionales de innovación, Universidad del País Vasco, Servicio de Publicaciones.

Olazaran, M. y Moso, M. (1999): "Social Studies of Science and Technology in Spain", en K. Sorensen (ed.): Similar Concerns, Different Styles? Technology Studies in Western Europe, vol. 2, COST-A4 Series, DG XII, Comisión Europea, Bruselas, pp. 61-96.

Sanz, L. (1998): "Socio-economic Studies of Science, Technology and Innovation in Spain", Easst Review, vol. 17, n. 4.
Sebastian, J. y Muñoz, E. (eds.) (2006): Radiografía de la investigación pública en España, Madrid, Biblioteca Nueva.

Sharif, N. (2006): "Emergence and development of the National Innovation Systems concept", Research Policy, vol. 35, n. 5, pp. 745-766.

Simmie, J. (2002): "Knowledge spillovers and reasons for the concentration of innovative SMEs", Urban Studies, vol. 39, n. $^{\text {s }} 5-6$, pp. 885-902.

Sternberg, R. (2000): "Innovation Networks and Regional Development-Evidence from the European Regional Innovation Survey (ERIS): Theoretical Concepts, Methodological Approach, Empirical Basis and Introduction to the Theme Issue", European Planning Studies, vol. 8, n. 4 , pp. 389-407.

Storper, M. (1997): The regional economy, New York, Guilford Press.

Tödtling, F. y Trippl, M. (2004): "Like phoenix from the ashes? The renewal of clusters in old industrial areas", Urban Studies, vol. 41, n. ${ }^{\circ 5} 5 / 6$, pp. 1175-1995.

Todtling, F. y Trippl, M. (2005): "One size fits all? Towards a differenciated regional innovation policy approach?", Research Policy, vol. 34, n. ${ }^{\circ}$ 8, pp. 1203-1219.

Vence, X. (2007): Crecimiento y políticas de innovación. Nuevas tendencias $y$ experiencias comparadas, Madrid, Pirámide.

Vickers, I. y North, D. (2000): "Regional technology Initiatives: Some insights from the English Regions", European Planning Studies, vol. 8, n. 3 , pp. 301318.

Whitley, R. (ed.) (1992): European Business Systems. Firms and Markets in their national contexts, London, Sage.

Whitley, R. (1999): Divergent Capitalisms. The social structuring and change of business systems, Oxford, Oxford University Press. 